\title{
FastqCleaner: an interactive Bioconductor application for quality-control, filtering and trimming of FASTQ files
}

\author{
Leandro Gabriel Roser D, Fernán Agüero and Daniel Oscar Sánchez
}

\begin{abstract}
Background: Exploration and processing of FASTQ files are the first steps in state-of-the-art data analysis workflows of Next Generation Sequencing (NGS) platforms. The large amount of data generated by these technologies has put a challenge in terms of rapid analysis and visualization of sequencing information. Recent integration of the $R$ data analysis platform with web visual frameworks has stimulated the development of user-friendly, powerful, and dynamic NGS data analysis applications.

Results: This paper presents FastqCleaner, a Bioconductor visual application for both quality-control (QC) and preprocessing of FASTQ files. The interface shows diagnostic information for the input and output data and allows to select a series of filtering and trimming operations in an interactive framework. FastaCleaner combines the technology of Bioconductor for NGS data analysis with the data visualization advantages of a web environment.

Conclusions: FastqCleaner is an user-friendly, offline-capable tool that enables access to advanced Bioconductor infrastructure. The novel concept of a Bioconductor interactive application that can be used without the need for programming skills, makes FastqCleaner a valuable resource for NGS data analysis.
\end{abstract}

Keywords: Bioconductor, FASTQ, Next generation sequencing, R, Shiny, User-friendly tool, Visualization, Web app

\section{Background}

The advent of Next Generation Sequencing (NGS) technologies has revolutionized genomics, transcriptomics and epigenomics research $[1,2]$. The large amount of genetic information produced by these instruments requires suitable data handling and exploration methods. For most common platforms, FASTQ files are the raw starting material for subsequent analyses. A portion of the reads can include adapters or contaminants, the quality of the sequences becomes generally lower towards the end of the reads, and ambiguous base calls may be present. The correction of these and other artifacts are important steps that should be performed before using sequencing reads for mapping or assembly.

Bioconductor [3] is a widely used repository based on the $\mathrm{R}$ programming language [4], containing tools devoted to the analysis of high-throughput genomic data. The massive use of these tools is, however, limited by the learning curve that users need to go through to work with customized code routines. Recently, $\mathrm{R}$ integration with web tools, in particular JavaScript APIs, has dramatically increased the potential of $\mathrm{R}$ to produce more interactive and dynamic experiences of data analysis. This integration is promissory to promote the adoption of $\mathrm{R}$ by many researchers for whom learning a programming language has proven to be a prohibitive investment of time and effort.

Here we present FastqCleaner, an R package with an offline-capable web application for QC, trimming and filtering of FASTQ files. The tool combines Bioconductor libraries for data analysis and the dynamism of a web application for data visualization.

\section{Implementation \\ Application overview \\ FastqCleaner offers the following features:}

1) Implementation of a local, offline-capable and userfriendly web interface.

2) Processing of Single-Read (SR) and Paired-End (PE) files. 
3) Dynamic analysis of the input and output files, for customizable sampling size of reads.

4) Interactive, dynamical exploration and visualization of the data, using cutting-edge technology based on JavaScript and CSS3.

5) Cross-platform (running in Linux, Mac-OSX and Windows).

6) Open source, under MIT license.

\section{Program architecture}

FastqCleaner was developed in $\mathrm{R}$ and is distributed as an $R$ package. Data processing is controlled via $R$ functions, that can be also accessed as normal functions from the $R$ console (Additional file 1). These programs make extensive use of the Bioconductor packages IRanges [5], Biostrings [6] and ShortRead [7]. For speed improvement of the routines, $\mathrm{C}++$ code was implemented in $\mathrm{R}$ using the Rcpp API [8]. The web interface included in the package was developed with Shiny [9], using JavaScript code written via the jQuery API, and CSS3.

\section{Design}

FastqCleaner takes compressed or uncompressed SR or PE files as input (Fig. 1). It accepts files with qualities in both Phred +33 and Phred +64 encoding, detecting Sanger, Solexa and Illumina $1.3+, 1.5+$, and $>1.8+$ formats.
Input files can be processed through a set of independent filters based on either one of the following two principles: 1) Remotion of a subset of reads that do not meet a given criterion. This group of filters can remove: a) reads with unknown bases (Ns), b) low complexity sequences, c) duplicated reads, d) reads with length below a threshold quality value, and e) reads with an average quality below a threshold value. 2) Trimming of individual reads. This group of filters can trim: a) full and partial adapters, b) 5' regions below a predefined quality threshold, and c) 3' or 5 ' regions for a fixed nucleotide length. The adapter trimming algorithm extends the methodology of the trimLRPatterns function of Biostrings, designed to trim on the flanks of reads. For this purpose, FastqCleaner includes the adapter_filter function, a wrapper of trimLRPatterns. The function is able to trim both adapters present on the flanks or within reads (Fig. 2). Several parameters can be passed to modify the behavior of the tool. These parameters allow, for example, to select a different threshold for the number of mismatches, to take into account the presence of indels, etc.

For SR files, FastqCleaner sequentially processes a block of reads and writes the resulting post-processed block into the corresponding output file. For PE files, the program uses in each cycle a two-step procedure: first, a block of forward and another of reverse reads are

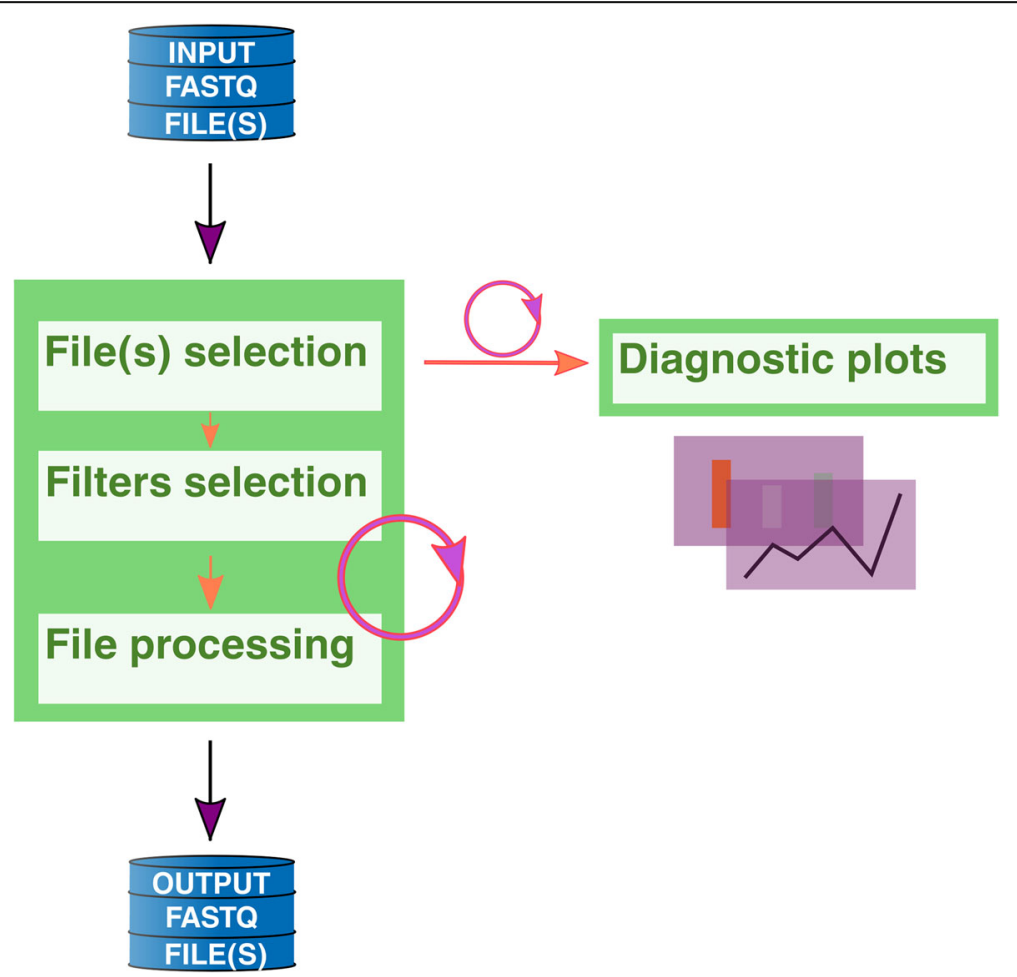

Fig. 1 Graphical representation of a typical workflow with FastqCleaner, showing the initial selection of FASTQ file(s), processing, and generation of output(s). Diagnostic interactive plots can be constructed for both input and output files. Circular arrows indicate halfway points in the workflow, where different configurations can be selected to re-run the program from there 


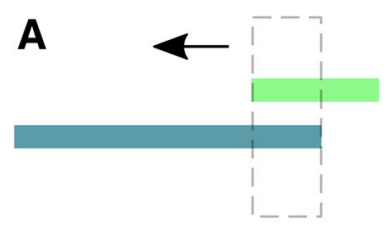

D

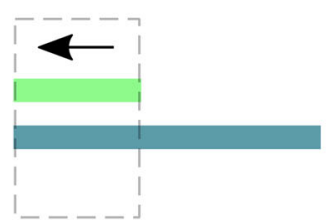

B

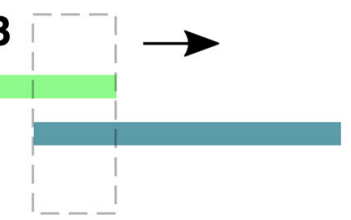

$\mathbf{E}$

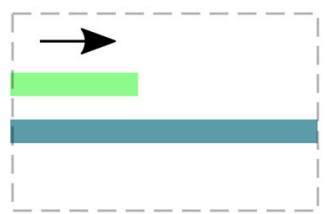

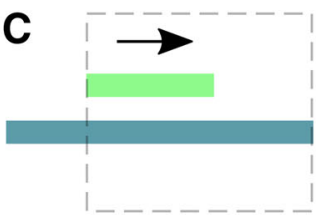

$\mathbf{F}$

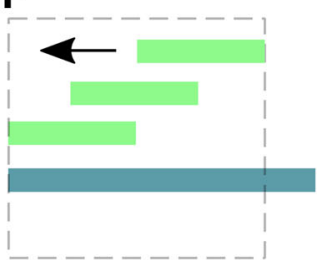

Fig. 2 Examples for adapter trimming. Pictures show the relative position of an adapter and a read, and the expected result after processing with the adapter_filter function of FastaCleaner. Dotted lines indicate the portion of the read that will be removed. Arrows show the direction along the read used for the program to seek for matches. If one or more matches are found, the function trims the longest subsequence, that contains the matching region plus the rest of the read, in the corresponding trimming direction. a partial adapter on the right + right-trimming of anchored adapter. b partial adapter on the left + left-trimming of anchored adapter. c partial adapter within read + right-trimming. D,E: full match between an adapter and a portion of the read + left- $(\mathbf{d})$ or right- (e) trimming. $\mathbf{f}$ multiple matches for a same adapter + left-trimming

separately processed as in the SR case, and then only those reads present in both post-processed blocks are written into the corresponding output files. Diagnostic plots are constructed using a random sample of reads with customizable size. This feature and the chunkwise processing methodology mentioned above, allow to work with relatively large files. The current version of FastqCleaner supports single-threaded processing. We will add multi-thread support in a future release of the package.

\section{Availability}

The application and a tutorial are available in Bioconductor at https://doi.org/doi:10.18129/B9.bioc.FastqCleaner. Source code for FastqCleaner is also available at GitHub (https://github.com/leandroroser/FastqCleaner) and in Additional file 3.

\section{Installation}

The application can be installed following the instructions detailed at https://doi.org/doi:10.18129/B9.bioc.FastqCleaner

\section{Launching the application}

The application can be launched with the following commands in the R console:

\section{library ("FastqCleaner") \\ launch_fqc()}

Optionally, when the application is used in RStudio (versions 0.99 .878 or higher), a button that allows the direct launch of the application with a single click can be found in the addins menu (Fig. 3).

\section{Results}

The web interface with its three main tabs is described in Fig. 4. The first tab (Fig. 4a) shows the file-selection menu, the available filters, and the run/reset buttons. The selection of files and filters represents the starting point in the FastqCleaner workflow. The second tab (Fig. 4b) shows the sequential operations performed on reads after processing. This information consists of the names of the input and output files, and a summary of informative statistics of the reads that passed the filter. The third tab (Figs. 4c, d) shows tables and interactive plots for data diagnostic. Plots can be constructed for both input (original data) and output (post-processed) files. A table with the most frequent $\mathrm{k}$-mers can also be visualized.

Analysis of SR pre-processing (Fig. 5) showed that the compared tools can be divided into three groups, in function of significant differences observed in processing speed for routine operations (Tukey HSD test, $p<0.001$ for all the three pairwise comparisons). The slowest were cutadapt and FASTX-Toolkit (group 1), while AdapterRemoval and Trimmomatic were the fastest (group 2). FastqCleaner showed an intermediate performance, comparable to Skewer and FLEXBAR (group 3).

Benchmarking of PE pre-processing operations (Fig. 6) showed that FastqCleaner significantly outperforms all other tools for routine operations (Tukey HSD test, $\mathrm{p}<$ 0.001 for pairwise comparisons of FastqCleaner versus each of the other applications). These results are valid for singlethreaded test conditions, and may vary when a multithread configuration is used with those programs supporting this feature. Simulated datasets specifically designed for adapter trimming, showed a similar performance in relation to other tools, with a slightly lower performance for some of the computed statistics (Table 1). 


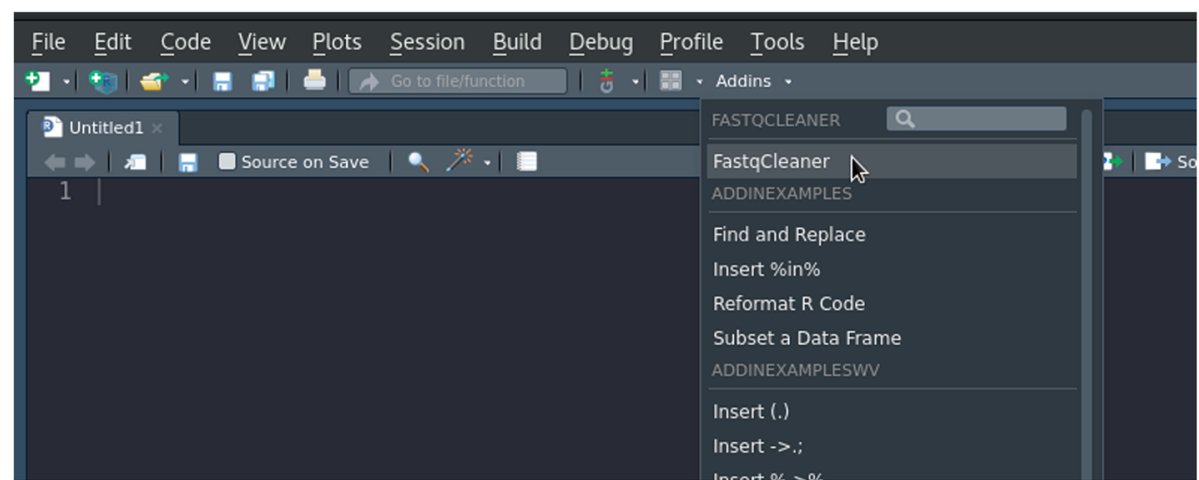

Fig. 3 RStudio addins menu, showing the button to launch the FastaCleaner application

\section{Discussion}

Benchmarking results for SR pre-processing indicated an excellent performance of FastqCleaner in comparison with other tools in terms of elapsed time. Benchmarking of PE pre-processing operations showed that FastqCleaner significantly outperforms all other compared tools for routine operations under standardized conditions. Analyses with simulated datasets designed for adapter trimming, also indicated a good overall performance of the application.

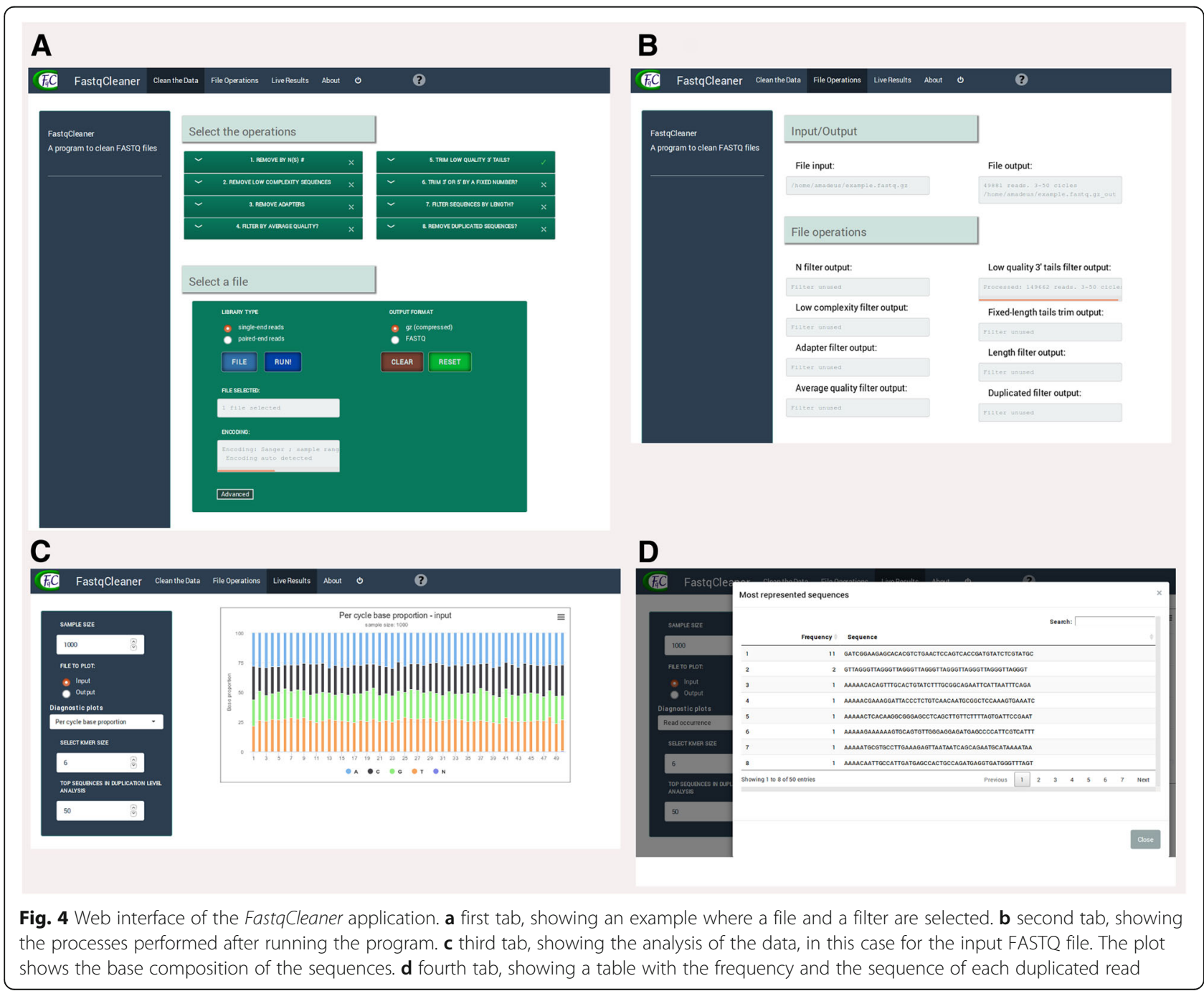




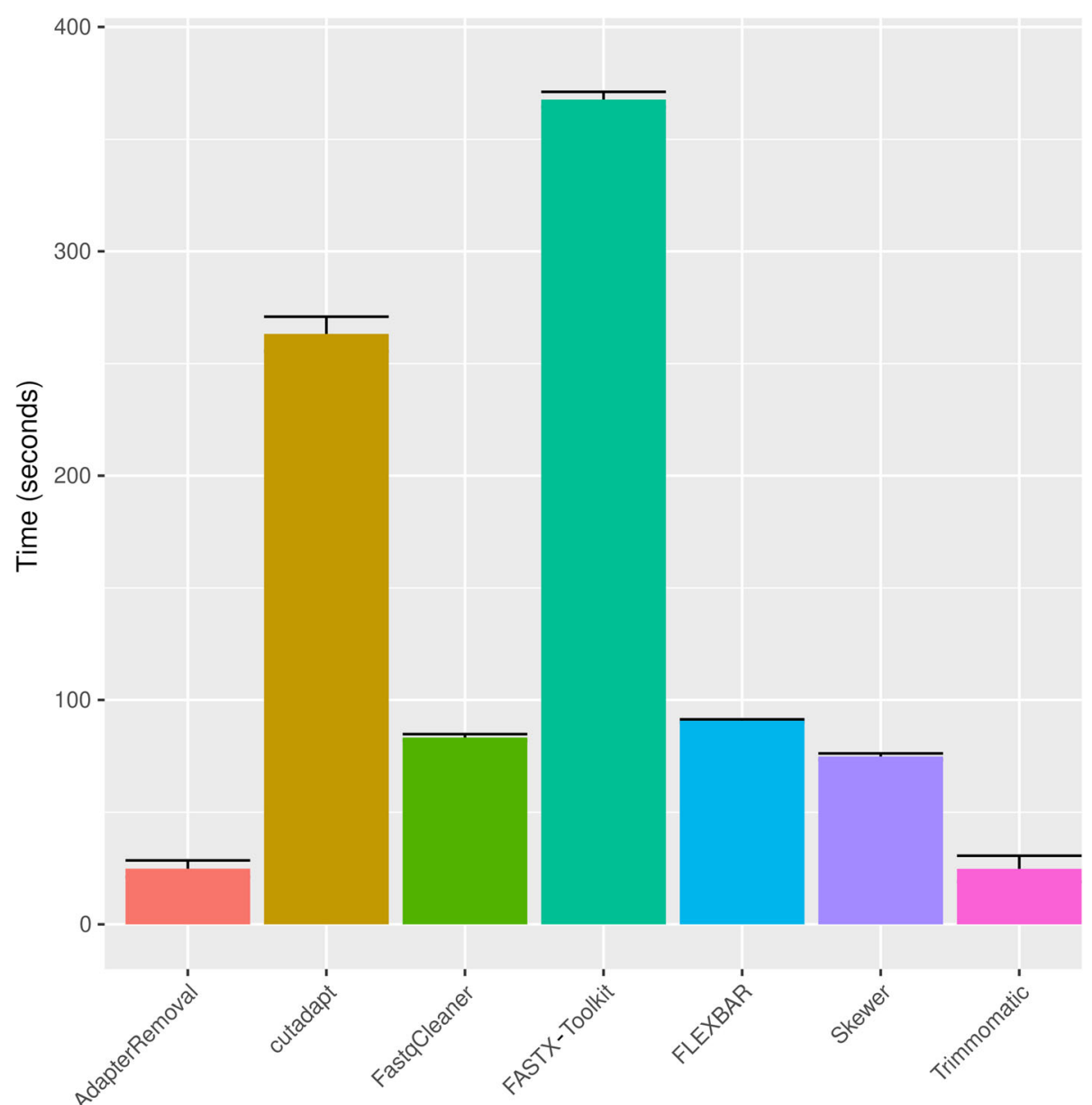

Fig. 5 Bar plot for elapsed time (in seconds) for SR adapter trimming and read length filtering

\section{Conclusions}

FastqCleaner is a tool with a rich and interactive cutting-edge graphical interface for pre-processing and exploration of SR and PE FASTQ files. Comparison with other available programs in a typical pre-processing scenario of adapter trimming and length filtering, showed an excellent performance of the application for both SR and PE real datasets. Simulated datasets designed for trimming operations showed comparable values of the performance statistics, with slightly lower values for some of them. The application is made available as an open source license. Coding experience is not required for its use, and is therefore particularly useful for users who are unfamiliar with $\mathrm{R}$ programming. Furthermore, all processing happens locally in the user's computer (even if the computer is disconnected from the network), making FastqCleaner amenable to run in environments where data confidentiality prevents uploading of files to the cloud.

In essence, FastqCleaner's dual capability facilitates both access to the underlying state-of-art Bioconductor infrastructure and to dynamic graphical visualizations in a $100 \%$ client-side friendly web environment. This makes FastqCleaner a novel technological advance for the analysis of Next Generation Sequencing data.

\section{Methods}

Experiments on actual data for overall evaluation of the application

In order to assess the performance of FastqCleaner, we have compared the package with other available preprocessing tools in benchmark tests: AdapterRemoval 2.2.2 [10], cutadapt 1.14 [11], FASTX-Toolkit 0.0.13 [12], FLEXBAR 3.0.3 [13], Skewer 0.2.2 [14] and Trimmomatic 0.36 [15]. The tests (Additional file 2) were conducted for adapter removal and length filtering using SR and PE files, with 22 replicates of each tests for statistical analysis of performance. Processing conditions were standardized by disabling compression of output files and using a single thread. In addition, pre-processing in FastqCleaner was performed using a chunk size of 10,000 reads per cycle. For SR processing, we downloaded from SRA the dataset SRR014966, with $14.3 \mathrm{M}$ reads of $36 \mathrm{bp}$. 


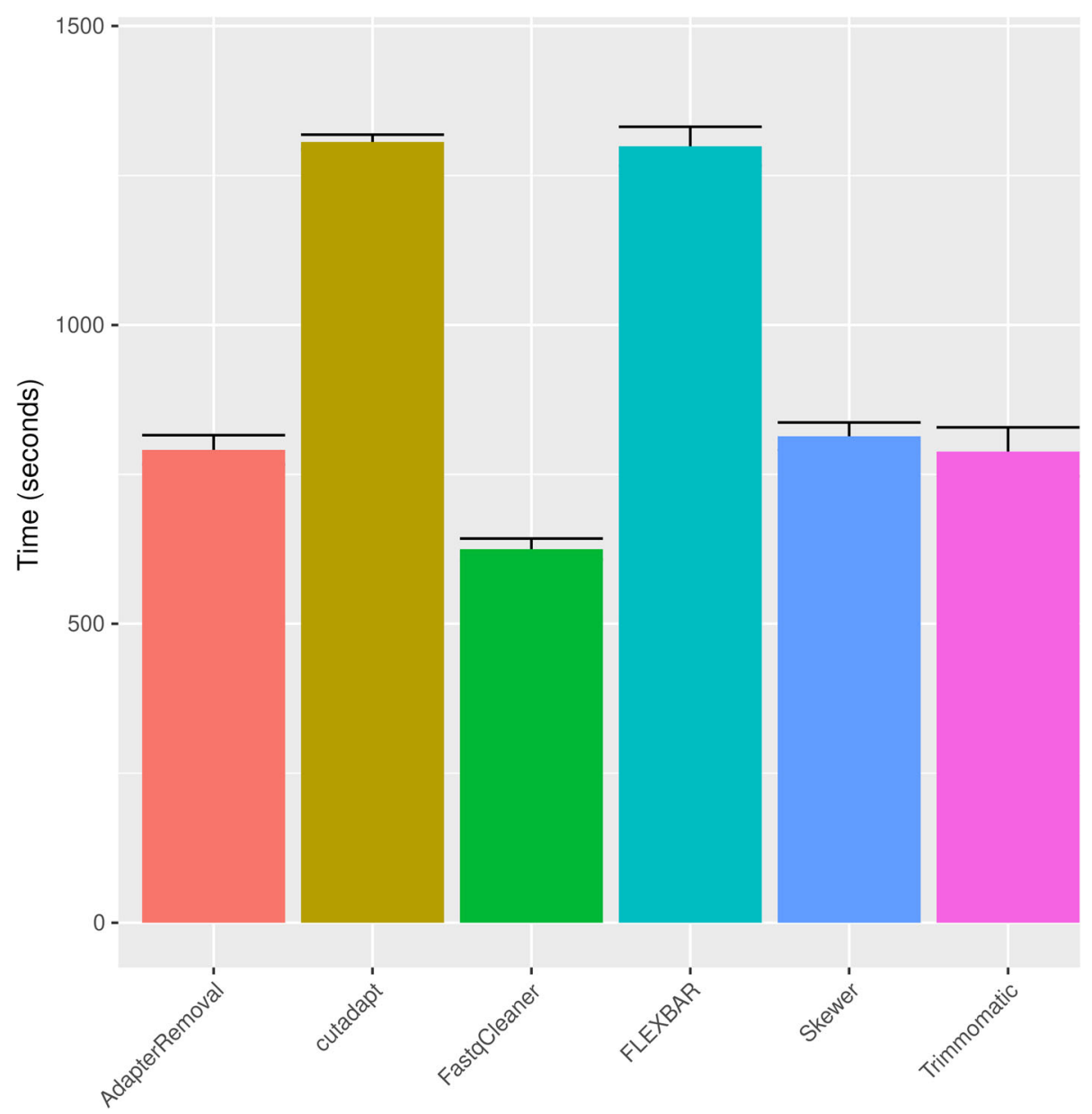

Fig. 6 Bar plot for elapsed time (in seconds) for PE adapter trimming and read length filtering. FASTX-Toolkit is not capable to process PE reads and is not shown in the plot

For PE processing, we downloaded the dataset SRR330569 with $27 \mathrm{M}$ reads of $101 \mathrm{bp}$. Benchmark tests were conducted in $\mathrm{R}$ using a laptop with Linux, a 2.20GHz Intel Core i7 CPU and $16 \mathrm{~GB}$ of $1600 \mathrm{MHz}$ RAM (Additional file 2).

\section{Experiments on simulated data for adapter trimming evaluation}

We simulated $100 \mathrm{bp}$ PE reads using the following conditions: fold coverage 10X, mean fragment length of 200

Table 1 Trimming statistics for simulated reads corresponding to the average results for mate 1 and mate 2

\begin{tabular}{llllll}
\hline & SEN & SPC & PPV & NVP & MCC \\
\hline AdapterRemoval & 0.999 & 1.000 & 1.000 & 0.999 & 0.999 \\
Skewer & 0.999 & 1.000 & 1.000 & 0.999 & 0.999 \\
cutadapt & 0.907 & 0.985 & 0.983 & 0.914 & 0.894 \\
FastaCleaner & 0.803 & 0.987 & 0.985 & 0.834 & 0.804 \\
\hline Acronyms:
\end{tabular}

Acronyms: sensitivity (SEN), specificity (SPC), positive predictive value (PPV), negative predictive value (NPV), and Matthews correlation coefficient (MCC) bp and standard deviation of 70, MiSeq2500 reads from the Escherichia coli O157:H7 strain (https://www.ncbi.nlm. nih.gov/nuccore/CP014314.1?report=fasta), and righttrimming. The simulations were performed using ART, a NGS read simulator [16]. We used a modified version adapted for simulating adapter-contaminated reads, available at https://sourceforge.net/projects/skewer/files/Simulator/. The trained profile was constructed using the ARTMountRainier version of art_profiler_illumina available at https://www.niehs.nih.gov/research/resources/software/biostatistics/art/index.cfm. This profile was from real Escherichia coli O157:H7 isolates (SRA dataset SRR957847). We compared FastqCleaner with other adapter trimming tools. For FastqCleaner, we used error rates between 0.1 and 0.2, averaging the resulting values of the statistics for final data presentation.

Trimming quality was assessed following Lindgreen [17] computing TP (True Positives), the proportion of contaminated reads trimmed to the actual known non-contaminated length, TN (True Negatives), the proportion of untrimmed 
non-contaminated reads, FP (False Positives), the proportion of over-trimmed reads, and FN (False Negatives), the proportion of under-trimmed reads. Using these four values, the following final statistics were computed: sensitivity [SEN = $\mathrm{TP} /(\mathrm{TP}+\mathrm{FN})]$, specificity $[\mathrm{SPC}=\mathrm{TN} /(\mathrm{FP}+\mathrm{TN})]$, positive predictive value $[\mathrm{PPV}=\mathrm{TP} /(\mathrm{TP}+\mathrm{FP})]$, negative predictive value $[\mathrm{NPV}=\mathrm{TN} /(\mathrm{TN}+\mathrm{FN})]$, and, as an overall performance measure, the Matthews correlation coefficient $\{\mathrm{MCC}=$ $(\mathrm{TP} \times \mathrm{TN}-\mathrm{FP} \times \mathrm{FN}) / \sqrt{ }[(\mathrm{TP}+\mathrm{FP}) \times(\mathrm{TP}+\mathrm{FN}) \times(\mathrm{TN}+\mathrm{FP})$ $\times(\mathrm{TN}+\mathrm{FN})]\}$. The "generate_data.R" script available as Additional file 4 contains the commands required to generate the simulations used for this part of the paper. The "generate_statistics.R" function available as Additional file 5 defines the code required to compute each of the statistics as per Lindgreen [17].

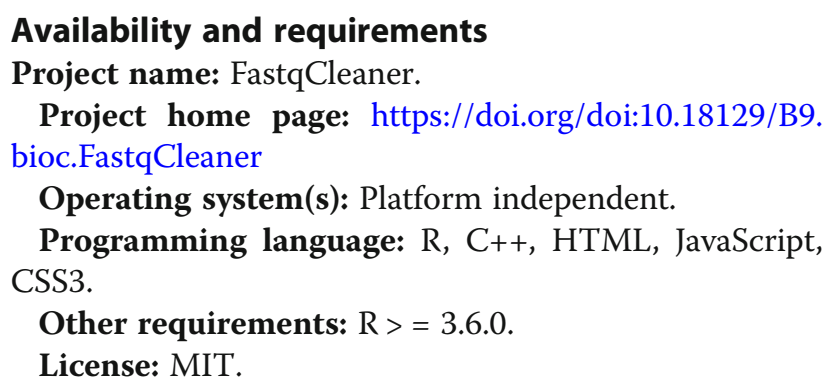

Any restrictions to use by non-academics: Not applicable.

\section{Additional files}

Additional file 1: PDF version of the online tutorial. (PDF $1048 \mathrm{~kb}$ )

Additional file 2: $\mathrm{R}$ script used in this work for benchmark testing. (R 3 $\mathrm{kb})$

Additional file 3: Source code of FastqCleaner. (GZ $3273 \mathrm{~kb}$ )

Additional file 4: $\mathrm{R}$ script to compute simulated data using ART for Escherichia coli O157:H7 data. (R 3 kb)

Additional file 5: $\mathrm{R}$ script to compute statistics for the different trimming tools as per Lindgreen (2017). (R 970 bytes)

\section{Abbreviations}

NGS: Next Generation Sequencing; PE: Paired-End Reads; SR: Single-Reads

\section{Acknowledgements}

FA and DOS are Career Investigators from CONICET (Consejo Nacional de Investigaciones Científicas y Técnicas, Argentina).

\section{Authors' contributions}

LGR designed and developed the R package. FA contributed to the improvement of the original design. LGR, FA and DOS wrote the manuscript and tested the package. DOS and FA supervised the project. All authors read and approved the final manuscript.

\section{Funding}

This work was supported by Agencia Nacional de Promoción Científica y Tecnológica (ANPCyT, Argentina. PICT-2014-0879 to DOS). The funding agency was not involved in the design, data analysis, interpretation of data, manuscript preparation, or publication design.

\section{Availability of data and materials}

FastaCleaner is freely available from its Bioconductor home page at https:// doi.org/doi:10.18129/B9.bioc.FastqCleaner under MIT license. FastaCleaner can be launched on any system that has R installed. An online tutorial is available at the package home page. A PDF version of this tutorial is included as supplemental material (Additional file 1). Source code for FastqCleaner is also available at GitHub (https://github.com/leandroroser/ FastqCleaner) and in Additional file 3.

Ethics approval and consent to participate

Not applicable.

\section{Consent for publication}

Not applicable.

\section{Competing interests}

The authors declare that they have no competing interests.

Received: 5 September 2018 Accepted: 20 June 2019

Published online: 28 June 2019

References

1. Koboldt D, Steinberg K, Larson D, Wilson R, Mardis E. The next-generation sequencing revolution and its impact on genomics. Cell. 2013;155:27-38.

2. Tripathi R, Sharma P, Chakraborty P, Varadwaj P. Next-generation sequencing revolution through big data analytics. Front Life Sci. 2016;9:11949.

3. Huber W, Carey V, Gentleman R, Anders S, Carlson M, Carvalho B, Bravo H, Davis S, Gatto L, Girke T, Gottardo R. Orchestrating high-throughput genomic analysis with Bioconductor. Nat Methods. 2015;12:115-21.

4. R Core Team. R: A Language and Environment for Statistical Computing Vienna: R Foundation for Statistical Computing; 2017.

5. Lawrence M, Huber W, Pages H, Aboyoun P, Carlson M, Gentleman R, Morgan M, Carey V. Software for computing and annotating genomic ranges. PLoS Comput Biol. 2013;9:e1003118.

6. Pagès $H$, Aboyoun $P$, Gentleman R, Deb Roy S. Biostrings: String objects representing biological sequences, and matching algorithms. $\mathrm{R}$ package version. 2017;2(44):2

7. Morgan M, Anders S, Lawrence M, Aboyoun P, Pages H, Gentleman R. ShortRead: a Bioconductor package for input, quality assessment and exploration of high-throughput sequence data. Bioinformatics. 2009;25: 2607-8

8. Eddelbuettel D, Romain F, Allaire J, Chambers J, Bates D, Ushey K. Rcpp: seamless R and C++ integration. J Stat Softw. 2011:40:1-18.

9. Chang W, Cheng J, Allaire JJ, Xie Y, MC Pherson J. Shiny: Web application framework for R. R package version 0.14.2. 2016. https://CRAN.R-project.org/ package $=$ shiny

10. Schubert M, Stinus L, Ludovic O. AdapterRemoval v2: rapid adapter trimming, identification, and read merging. BMC R Notes. 2016;9:88.

11. Martin M. Cutadapt removes adapter sequences from high-throughput sequencing reads. EMBnet J. 2011;17:10.

12. Hannon G. FASTX Toolkit. http://hannonlab.cshl.edu/fastx_toolkit/index.html. Accessed 24 June 2019

13. Dodt M, Roehr J, Ahmed R, Dieterich C. FLEXBAR — flexible barcode and adapter processing for next-generation sequencing platforms. Biology. 2012; 1:895-905.

14. Jiang $H$, Lei $R$, Ding SW, Zhu S. Skewer: a fast and accurate adapter trimmer for next-generation sequencing paired-end reads. BMC bioinformatics. 2014; 15:182.

15. Bolger A, Lohse M, Usadel B. Trimmomatic: a flexible trimmer for Illumina sequence data. Bioinformatics. 2014:30:2114-20.

16. Huang W, Li L, Myers JR, Marth GT. Art: a next-generation sequencing read simulator. Bioinformatics. 2012;28:593-4.

17. Lindgreen S. Adapterremoval: easy cleaning of next-generation sequencing reads. BMC Res Notes. 2012:5:337.

\section{Publisher's Note}

Springer Nature remains neutral with regard to jurisdictional claims in published maps and institutional affiliations. 